\title{
Exercício físico como instrumento para a melhoria da respiração na doença de Parkinson, um estudo de caso
}

\author{
Luciana Renata Conceição*, Kátia Tanaka**
}

\section{Resumo}

A doença de Parkinson (DP) é uma enfermidade crônica e degenerativa que acomete, principalmente, as funções motoras. Os sinais e sintomas característicos da doença são tremor, acinesia, rigidez muscular e instabilidade postural. Devido à rigidez que leva ao comprometimento dos músculos flexores do tronco, o indivíduo com DP passa a apresentar diminuição da capacidade respiratória. Assim, o objetivo do presente estudo é analisar os efeitos do exercício físico e respiratório sobre aspectos físicos, respiratórios e cognitivos na DP. Trata-se de um estudo de caso, com duração de quatro meses e coleta de dados pré, intermédio e pós-intervenção. O voluntário foi um indivíduo do sexo masculino que tinha como queixa principal a dificuldade respiratória, além de ter diagnóstico clínico com DP idiopática, estágio da doença entre leve e moderado, sem indício de demência e nível de atividade física considerado como ativo. Inicialmente foram realizadas anamnese e avaliação clínica da doença; em seguida, avaliou-se a cognição e as capacidades físicas e respiratórias. A intervenção consistiu em exercícios físicos que o participante já estava executando, aos quais foi dada continuidade de forma progressiva e acrescentados exercícios respiratórios durante dois meses, duas vezes por semana, com duração de uma hora por sessão. Após esse prazo, foi realizada a reavaliação, utilizando-se o mesmo protocolo. Foram realizados mais dois meses de intervenção e, ao final, a reavaliação, o que totalizou os quatro meses de programa de exercício físico e respiratório. A análise dos dados foi feita qualitativa (clínica) e quantitativamente (porcentagem).

Palavras-chave: Doença de Parkinson. Respiração. Rigidez muscular. Exercício físico.

\section{Introdução}

A doença de Parkinson (DP) é uma enfermidade crônica e progressiva que acomete um em cada mil indivíduos da população em geral, afetando a qualidade de vida nos aspectos físico, mental, emocional, social e econômico (CAMAR-

* Especialista em Atividade Física para Grupos Especiais, Fundação Hermínio Ometto - Uniararas. Endereço para correspondência: Av. Dr. Maximiliano Baruto, 500, Jd. Universitário, Araras, SP. Brasil. CEP 13607339. E-mail: lucianarenatac@yahoo.com.br

** Doutora em Ciências pela Universidade Federal de São Paulo. Professora da Uniesp. E-mail: katiatanak@ gmail.com

$\rightarrow$ http://dx.doi.org/10.5335/rbceh.v12i2.4927

Recebido em: 07/04/2015. Aceito em: 18/08/2015 
GOS et al., 2004; SANTOS; SOUZA; BASTOS, 2007).

Isto acontece devido à degeneração da substância negra, que reduz a atividade dopaminérgica, afetando o funcionamento nigroestriatal e da região pré-frontal, o que caracteriza o comprometimento motor. As vias colinérgicas também são afetadas, especificamente o núcleo basal de Meynert, levando a um declínio cognitivo (DUJARDIN; LAURENT, 2003; TEKIN; CUMMINGS, 2002).

Os principais sinais e sintomas que acometem as pessoas com DP são acinesia, rigidez, tremor, instabilidade postural, além de problemas não motores, como memória, funções executivas, depressão e ansiedade (TANAKA, 2008).

Outra disfunção que se apresenta em grande parte das pessoas com DP é a respiratória. Segundo Pereira e Cardoso (2000), a DP pode causar diversas dificuldades em nível respiratório, comprometendo a funcionalidade da respiração e tornando debilitadas as trocas gasosas, além de reduzir os volumes, a capacidade e os fluxos pulmonares.

Os problemas respiratórios estão associados à rigidez muscular, principalmente na musculatura flexora, o que determina alterações de postura, como anteroflexão do tronco que é característico na DP (BARBOSA, 1991).

De acordo com O'Sullivan e Schimitz (1993), as complicações referentes à capacidade respiratória do indivíduo parkinsoniano devem-se a uma redução na capacidade de expansão torácica em decorrência da rigidez dos músculos intercostais e das posições de flexão e flexão-adução do tronco e membros supe- riores. Em estágios avançados, tal comprometimento respiratório é responsável por casos substanciais de mortalidade e morbidade (WERMUTH et al., 1995).

Além disso, esses distúrbios posturais levam a alterações na movimentação da articulação temporomandibular, que tem importante função na fonação (CARRO et al., 2001; SCHULZ; GRANT, 2000).

Uma das formas de amenizar os problemas e agravamentos provocados pela DP pode ser por meio de exercício físico e respiratório. Nos últimos anos, muitos estudos apresentaram os benefícios do exercício físico na DP (ABRANTES et al., 2012; CRUISE et al., 2011; TANAKA et al., 2009; GOBBI et al., 2009). Essa prática é utilizada como um tratamento não farmacológico a fim de propiciar melhoras na qualidade de vida do indivíduo (VITÓRIO et al., 2011; BARBIERI et al., 2012).

No entanto, poucos estudos relatam a influência do exercício físico na capacidade respiratória de pessoas com DP. Em outros grupos, o exercício físico pode melhorar a captação, o transporte e a utilização de oxigênio, tornando o sistema respiratório mais eficiente (SOCIEDADE BRASILEIRA DE CARDIOLOGIA, 2005).

Segundo O'Sullivan e Schmitz (1993), os exercícios de alongamento e resistência da musculatura intercostal, combinados com exercícios respiratórios, podem aumentar a expansão da caixa torácica. Assim, nesta pesquisa, busca-se verificar a eficácia de tais exercícios físicos e respiratórios como procedimento reabilitativo alternativo à $\mathrm{DP}$, a fim de encontrar meios de auxiliar os parkinso- 
nianos na melhoria/manutenção de sua capacidade respiratória.

Desse modo, o objetivo do estudo é analisar os efeitos do exercício físico combinado ao exercício respiratório sobre os aspectos motores, a capacidade respiratória e a função cognitiva em um indivíduo com doença de Parkinson. Paralelamente, busca-se auxiliar os demais profissionais da área em sua atuação com as pessoas ou pacientes com Parkinson, podendo, inclusive, inserir em seus treinamentos os exercícios respiratórios que aqui foram listados.

\section{Material e métodos}

A pesquisa foi realizada por meio de um delineamento longitudinal (avaliação pré, intermédio e pós-treinamento), sendo um estudo de caso com duração de quatro meses; aprovado pelo Comitê de Ética em Pesquisa da instituição sob o Protocolo no 24997013.3.0000.5385. Foi apresentado ao participante um termo de consentimento livre e esclarecido que, concordando em participar, foi assinado. $\mathrm{O}$ estudo de caso foi realizado em uma academia de Rio Claro, em São Paulo. $\mathrm{O}$ voluntário da pesquisa apresentava diagnóstico clínico da DP idiopática, e sua principal queixa foi a diminuição da capacidade respiratória.

Como procedimento de estudo, primeiramente, fez-se uma anamnese com o histórico pessoal do indivíduo. Em seguida, o participante foi avaliado pelo seguinte protocolo:

a) avaliação clínica da doença: escala de Hoehn-Yahr modificada (HOEHN; YAHR, 1967), para avaliar o estagiamento da doença; b) avaliação física: questionário Baecke modificado para idosos (QBMI) (VOORRIPS et al., 1991), que quantifica o nível de atividade física dos idosos; Escala de Severidade de Fadiga (FSS) (HERLOFSON; LARSEN, 2002), para identificar a taxa de gravidade de fadiga, e Escala de Equilíbrio Funcional de Berg (BERG et al., 1992), realizada para avaliar o equilíbrio funcional do indivíduo;

c) avaliação respiratória: cirtometria (CALDEIRA et al., 2007), que determina o índice de amplitude (IA) de expansão torácica durante a respiração, e espirometria (PEREIRA, 2002), para prevenir e diagnosticar possíveis problemas respiratórios no voluntário;

d) avaliação cognitiva: o miniexame do estado mental (MEEM) (FOLSTEIN; FOLSTEIN; MCHUGH, 1975) indica o nível de comprometimento cognitivo do participante; o teste de trilhas A e B (Trail Making Test - TMT) (SPREEN; STRAUSS, 1998) avalia atenção, flexibilidade mental e procura visual; o miniquestionário do sono (Mini-Sleep Questionnairie) (ALÓE et al., 2000) investiga a qualidade do sono e a frequência das queixas do indivíduo; o inventário de ansiedade traço-estado (Idate) (GORENSTEIN; ANDRADE, 1996) verifica os traços de ansiedade e o estado de ansiedade; e a escala hospitalar de ansiedade e depressão (HAD) (MONDOLO et al., 2006) verifica a presença de sintomas depressivos e de ansiedade. 
Após a avaliação inicial, o participante continuou seu treinamento físico, que já vinha sendo desenvolvido, ao qual foi acrescido um programa de exercício respiratório. O treinamento físico foi realizado duas vezes por semana, 45 minutos por sessão, sendo distribuído da seguinte forma:

a) aquecimento na esteira;

b) alongamentos dinâmicos para os grandes grupos musculares;

c) exercícios musculares em suspensão, desenvolvidos em isometria, sendo duas séries de 20" para cada movimento, trabalhando músculos peitorais, dorsais, bíceps e tríceps braquial, posteriores da coxa e abdominais;

d) exercícios musculares localizados, utilizando-se de halteres e caneleiras, abrangendo a musculatura peitoral, dorsal, bíceps e tríceps braquial, quadríceps, posteriores, adutores e abdutores da coxa, executando-se duas séries com quinze repetições em cada exercício.

Já o programa de exercício respiratório contemplava seis exercícios, que levaram em torno de 15 minutos para serem desenvolvidos:

a) respiração baixa, estimulando a região abdominal;

b) respiração alta, exercitando a região torácica;

c) respiração média, trabalhando a região das últimas costelas;

d) respiração alternada: inspiração normal e expiração alternada das narinas; e) respiração pulmonar: inspiração máxima pelo nariz, seguida de uma pausa, tentando, logo após, inspirar mais ar, liberando-o depois pela expiração;

f) respiração de expansão: inspiração fracionada (inspiração seguida de pausa, quatro vezes) antes de expirar.

A combinação do exercício físico com o respiratório totalizou uma hora de duração. Após dois meses de treinamento, período que corresponde à metade da intervenção, realizaram-se avaliações para verificar possíveis benefícios do programa em curto período de tempo. $\mathrm{O}$ protocolo de treinamento estendeu-se por mais dois meses, concluindo quatro meses, o participante foi reavaliado pelo mesmo protocolo.

As avaliações foram realizadas no estado $O n$ (pico de ação) da medicação do participante. Como se trata de um estudo de caso, para a análise dos dados, os resultados foram apresentados na íntegra.

\section{Resultados e discussão}

Como já foi exposto, este trabalho visou analisar o efeito combinado de exercício físico e respiratório em um indivíduo acometido pela doença de $\mathrm{Pa}$ rkinson. Diversas são as origens dessa deficiência respiratória ocasionada na DP, como flexão de tronco e cifoescoliose, que juntamente com uma acentuada rigidez muscular, artrose e bradicinesia dificultam a expansão torácica, restringindo a passagem do ar.

RBCEH, Passo Fundo, v. 12, n. 2, p. I59- I7I, maio/ago. 2015 
Sabe-se que a prática de exercício físico de forma isolada pode trazer benefícios às pessoas com DP nos aspectos motores (BARBIERI et al., 2012; VITÓRIO et al., 2011), cognitivos (HOPKINS et al., 2012; TANAKA et al., 2009) e respiratórios (ALVES et al., 2005). Porém, a combinação de exercício físico e respiratório ainda é pouco estudada. Dessa forma, apresentam-se os resultados da presente pesquisa em termos dos aspectos motor, respiratório e cognitivo.

\section{Aspecto motor}

Em relação à avaliação do estagiamento da doença pela escala de Hoehn-Yahr, o resultado foi igual nas três fases, observando uma estabilidade no quadro clínico do indivíduo (Tabela 1). Levando-se em consideração que a doença aqui mencionada é neurodegenerativa, pode-se notar um benefício em conseguir manter o indivíduo no mesmo estágio da DP durante o período de pesquisa.

Já ao nível de atividade física, verificou-se que houve um declínio (Tabela 1). Optou-se por avaliar o nível de atividade física para controle do exercício proposto na pesquisa. Porém, esse questionário também leva em consideração atividades de vida diária, como lavar louças, e de lazer. Dessa forma, embora o participante tenha mantido sua prática de exercício físico e respiratório proposto pela pesquisa, ele reduziu as atividades que realizava em seu tempo livre, como pescaria, jardinagem e natação, o que interferiu nos resultados.
Tabela 1 - Resultados obtidos nas avaliações motoras do participante

\begin{tabular}{l|r|r|r}
\hline \multicolumn{1}{c|}{ Avaliações } & \multicolumn{1}{c|}{ Pré } & Intermédio & \multicolumn{1}{c}{ Pós } \\
\hline Estágio da doença & 2,5 & 2,5 & 2,5 \\
Nível de atividade física & 15,777 & 15,152 & 10,225 \\
Fadiga & 39 & 77 & 48 \\
Equilíbrio & 55 & 56 & 55 \\
\hline
\end{tabular}

Fonte: dos autores.

Nota: Estagiamento da doença pela escala de Hoehn-Yahr (em pontos); nível de atividade física pelo questionário Baecke modificado para idosos (em pontos); fadiga pela escala de severidade de fadiga (em pontos); equilíbrio pela escala de equilíbrio funcional de Berg (em pontos).

A fadiga, definida como estado de cansaço extremo, fraqueza ou falta de energia, é muito comum na DP (BROWN et al., 2005). Estudos apontam que entre $45 \%$ e $50 \%$ dos pacientes se queixam desse sintoma (GRACE; MENDELSOHN; FRIEDMAN, 2007).

No presente estudo, os resultados apontam um aumento no quadro de fadiga desde a avaliação inicial até a final, o que é esperado pela doença (Tabela 1). A escala utilizada, a escala de severidade de fadiga, é um questionário em que, no máximo, pode se atingir 63 pontos. No entanto, a nota de corte é de 36 pontos, acima disso, indica-se um quadro de fadiga no avaliado.

Nota-se, também, um aumento dessa fadiga na avaliação intermediária e uma redução na avaliação final, aproximando-se dos valores da avaliação inicial. A possível razão para esse resultado deve-se ao fato de o voluntário estar em uma fase de aumento de volume de exercício, uma vez que foi introduzida uma nova atividade em sua rotina de treinamento (respiratória), bem como aumento da so- 
brecarga de trabalho, pois há a intenção de se aposentar em breve. Passada essa fase e adaptando-se a atividade respiratória em seu programa, verificou-se na avaliação final uma redução da fadiga.

Sobre o equilíbrio, na DP verifica-se que distúrbios manifestam-se em diferentes estágios da patologia. Uma das causas deve-se à instabilidade postural apresentada como um dos sinais da doença (GRIMBERGEN et al., 2009). A instabilidade postural caracteriza-se pela perda dos reflexos de readaptação postural, o que leva a quedas frequentes (BARBOSA, 1991).

O exercício físico como treinamento resistido, por exemplo, tem apresentado benefícios na marcha e no equilíbrio de pessoas com DP (BRAGA et al., 2002).

Em relação à combinação entre exercício físico e respiratório, os resultados obtidos no presente estudo apontam uma melhora no desempenho do voluntário no teste de equilíbrio (Tabela 1 ). Na escala de equilíbrio funcional de Berg, a pontuação máxima é de 56 pontos, e quanto mais altos os resultados obtidos, melhor o equilíbrio do voluntário, que atingiu, respectivamente, escore 55, 56, 55 nas avaliações pré, durante e pós-intervenção.

\section{Aspecto respiratório}

A cirtometria foi realizada nos três momentos do estudo, juntamente com os outros testes; já a espirometria foi realizada apenas duas vezes: a primeira antes do início das intervenções e a segunda ao final.

Para a cirtometria, três pontos anatômicos foram utilizados: altura da prega axilar, região do apêndice xifoide e linha da cicatriz umbilical. O objetivo da cirtometria é encontrar o índice de amplitude (IA) de cada um dos pontos torácicos. A Tabela 2 apresenta os resultados da avaliação.

Tabela 2 - Resultados nas avaliações cirtométricas (expressos em cm)

\begin{tabular}{l|c|c|c|r|r|r|r|r|r}
\hline \multirow{2}{*}{ Tempo } & \multicolumn{2}{|c|}{ Prega axilar } & \multirow{2}{*}{ IA } & \multicolumn{2}{|c|}{ Apêndice xifoide } & \multirow{2}{*}{ IA } & \multicolumn{2}{|c|}{ Cicatriz umbilical } & \multirow{2}{*}{ IA } \\
\cline { 2 - 3 } & Insp. & Exp. & & Insp. & Exp. & & Insp. & \multicolumn{1}{c|}{ Exp. } & \\
\hline Pré & 110,5 & 105,0 & 5,5 & 106,5 & 95,5 & 11,0 & 103,0 & 99,5 & 3,5 \\
Intermédio & 111,0 & 105,0 & 6,0 & 103,5 & 95,5 & 8,0 & 104,5 & 101,0 & 3,5 \\
Pós & 113,0 & 108,0 & 5,0 & 106,5 & 96,0 & 10,5 & 103,0 & 101,5 & 1,5 \\
\hline
\end{tabular}

Fonte: dos autores.

Nota: IA: índice de amplitude; Insp.: inspiração; Exp.: expiração.

Os resultados do IA na última avaliação não variaram muito desde o início das coletas. Acreditava-se que, com a prática regular dos exercícios, os resultados seriam superiores aos iniciais. Contudo, ao analisar o questionário Baecke modificado para idosos (QBMI) (Tabela
1), percebe-se que o indivíduo diminuiu a prática de exercícios físicos (natação), atividade que vinha desenvolvendo paralelamente à pesquisa. No início do estudo, ele praticava três tipos de exercícios, mas, durante o período da pesquisa, acabou optando por praticar somente os 
exercícios contidos neste protocolo de treinamento. Acredita-se que isso tenha influenciado negativamente os resultados da última análise. Essa diferença tornou-se evidente também no laudo do exame de espirometria, que é um teste eficiente para medir a quantidade de ar que entra e sai dos pulmões; na avaliação pré-intervenção, o Best Pre FVC (melhor capacidade vital forçada - volume máximo de ar exalado com esforço máximo, a partir do ponto de máxima inspiração) atingiu 17,550 litros e o Best Pre FEV1 (melhor volume expiratório forçado no $1^{\circ}$ segundo) (PEREIRA, 2002) chegou aos 12,692 litros. No entanto, na última avaliação, o Best Pre FVC foi de 16,388 litros e o Best Pre FEV1 alcançou 11,398 litros. Assim, o laudo da primeira espirometria apontou exame normal, enquanto o segundo laudo situou o indivíduo como limítrofe de obstrução das vias aéreas.

Os problemas respiratórios são muito comuns e considerados a principal causa de morte nessa patologia (HOVESTADT et al., 1989). As causas estão relacionadas à diminuição da flexibilidade da musculatura responsável pela respira- ção, assim como as alterações posturais e a ativação da musculatura responsável por coordenar as vias aéreas (SABATÉ et al., 1996). Outra hipótese pode estar relacionada ao processo fisiológico da doença: a deficiência de dopamina, que também causa diminuição do controle da resposta ventilatória, alterando a sensibilidade ou a percepção à hipóxia (baixa concentração de oxigênio) (SEREBROVSKAYA et al., 1998).

Embora não tenha se observado uma melhora nos resultados das variáveis respiratórias, nos aspectos motores e cognitivos, os resultados foram satisfatórios, como podemos observar na descrição dos resultados dos testes que segue.

\section{Aspecto cognitivo}

O miniexame do estado mental foi a ferramenta utilizada para realizar o rastreio cognitivo, com a finalidade de identificar possível demência. $\mathrm{O}$ voluntário alcançou 29 pontos nas três avaliações, indicando que não se encontra em estado de demência, não havendo comprometimento cognitivo (Tabela 3 ).

Tabela 3 - Resultados obtidos nas avaliações cognitivas do participante

\begin{tabular}{l|r|r|r}
\hline \multicolumn{1}{c|}{ Avaliações } & Pré & Intermédio & \multicolumn{2}{|c}{ Pós } \\
\hline Rastreio cognitivo & 29 & 29 & 29 \\
Atenção e flexibilidade mental & TA=40" / TB=52" & TA=28" / TB=38” & TA=23" / TB=32" \\
Sono & 49 & 39 & 49 \\
Ansiedade-traço & 45 & 47 & 50 \\
Ansiedade-estado & 47 & 42 & 47 \\
Ansiedade e sintomas depressivos & $\mathrm{A}=6 / \mathrm{D}=8$ & $\mathrm{~A}=6 / \mathrm{D}=7$ & $\mathrm{~A}=12 / \mathrm{D}=8$ \\
\hline
\end{tabular}

Fonte: dos autores.

Nota: A: ansiedade; D: depressão; TA: teste de trilhas A; TB: teste de trilhas B. Rastreio cognitivo pelo miniexame do estado mental (em pontos); atenção e flexibilidade mental pelo teste de trilhas A e B (em segundos); sono pelo miniquestionário do sono; ansiedade pelo inventário de ansiedade traço-estado (Idate) (em pontos); ansiedade e sintomas depressivos pela escala hospitalar de ansiedade e depressão (HAD) (em pontos). 
Detalhando os dados coletados, compreende-se melhor cada resultado. $\mathrm{O}$ desempenho no teste de trilhas foi considerado muito bom. A média é de 40 " para o teste A e 75" para o B. O tempo gasto durante o teste é considerado deficiente quando é > 78" para a trilha A e > 273" para a B. Porém, nosso participante atingiu, na primeira avaliação, 40 " e 52" para A e B, respectivamente. Já na segunda avaliação, suas marcas chegaram a 28 " e 38", enquanto na última medição ele conseguiu realizar a tarefa em apenas 23 " e 32 ".

O miniquestionário do sono consiste em um conjunto de dez perguntas que identifica se a pessoa tem sono tranquilo ou não. Nessas questões, o participante pode alcançar no mínimo 10 e no máximo 70 pontos, em uma escala que divide 0 escore da seguinte forma: 10-24 pontos, sono bom; $25-27$ pontos, sono levemente alterado; 28-30 pontos, sono moderadamente alterado; acima de 30 pontos, sono muito alterado. Dentro desse panorama, percebe-se que o participante possui alteração acentuada no sono nas três avaliações, apresentando pequena melhora na qualidade na avaliação intermediária, mas ainda dentro do escore muito alterado.

$\mathrm{O}$ questionário que avalia a ansiedade (Idate - traço/estado) é composto de duas partes. A parte referente ao traço verifica o nível de ansiedade que o indivíduo geralmente sente, ou seja, característico de sua personalidade. Já a parte que se refere ao estado, verifica a ansiedade no momento. Assim, observando os resultados nos três momentos e das duas partes do teste, nota-se que o participante apresenta uma caracte- rística ansiosa e que não sofre muitas alterações no decorrer da pesquisa.

$\mathrm{Na}$ escala hospitalar de ansiedade e depressão (HAD), podemos observar que o quadro de depressão manteve-se estável em todas as avaliações, que foi de 6 pontos nas duas primeiras avaliações e 12 na terceira. Provavelmente, isso se deve ao fato de o indivíduo ser naturalmente ansioso, como observado no Idate traço, já mencionado.

Tanto a prática de exercício físico quanto a de exercício respiratório podem ser benéficas para a DP, bem como para a cognição, a ansiedade, os sintomas depressivos e o sono (GOBBI et al., 2014; ROSA; CIELO; CECHELLA, 2009).

Comparando-se os resultados obtidos com a literatura (GONÇALVES, 2007; ROSA; CIELO; CECHELLA, 2009), observa-se que a prática de exercícios respiratórios regulares é capaz de atuar também nos sintomas secundários da doença de Parkinson. Muotri, Nunes e Bernik (2007) encontraram, em sua revisão de literatura, que tais exercícios atuam de maneira positiva no tratamento de transtornos ansiosos, devido à sua ação no controle respiratório dos indivíduos.

À mesma conclusão chegou Neves Neto (2011) em seu estudo, no qual verificou que os níveis de depressão, ansiedade e insônia diminuíram com a prática de exercícios respiratórios. No presente estudo, também se pode observar discretas melhoras, como diminuição dos sintomas depressivos e melhora no padrão do sono. Para Cole (2005), a prática de exercício respiratório pode ser considerada uma técnica não farmacológica para benefícios do sono e da cognição. 
Em estudo de Gonçalves (2007), além de se observar resultados também nessas variáveis, notou-se melhorias inclusive no aspecto cognitivo, sobretudo na memória episódica de idosos saudáveis, achado que King et al. (2007) também constataram em sua pesquisa, a qual relacionou os exercícios respiratórios em pessoas com transtorno do pânico. Pode-se considerar que, no presente estudo, o resultado também foi positivo, visto que o voluntário manteve o mesmo escore em seu miniexame do estado mental (MEEM) nas três avaliações, e que a DP é uma doença neurodegenerativa progressiva, portanto, é até mesmo esperada sua evolução.

Para as pessoas com DP, participar de programas de exercício físico e/ou respiratório pode melhorar a qualidade de vida, mantendo sua independência motora, que é importante para as atividades de vida diária (COELHO et al., 2013; GONÇALVES, 2007; PARREIRA, 2003). Em uma situação inversa, de inatividade física, o processo pode se agravar, piorando o quadro clínico da DP, comprometendo a funcionalidade, levando a quedas, ao acamamento e à hospitalização (TANAKA, 2009). A simples manutenção dos resultados das variáveis analisadas no presente estudo pode mostrar benefícios à qualidade de vida do participante.

\section{Considerações finais}

No presente trabalho, que teve como objetivo principal analisar os efeitos do exercício físico combinado ao exercício respiratório sobre os aspectos físicos, respiratórios e cognitivos na doença de Parkinson, verifica-se que, mesmo sendo uma doença neurodegenerativa, a prática de tal protocolo foi eficaz para o voluntário que, com o avanço do treinamento, mostrava-se cada vez mais aplicado em realizar os exercícios, pois conseguia sentir em sua rotina que sua aptidão física e respiratória estava maior.

É importante também destacar que o participante da pesquisa sempre treinou em condições favoráveis, não sofrendo pressão alguma para a execução dos exercícios e realizando-os de acordo com suas possibilidades, fato que propiciou um ambiente adequado para o desenvolvimento das tarefas. Outro aspecto relevante é que suas condições diárias sempre foram respeitadas, ou seja, quando ele não se julgava apto naquele dia a fazer determinado exercício, era encorajado a tentar, mas, persistindo em sua negação, sua decisão era respeitada.

Em outro aspecto, no decorrer do levantamento bibliográfico sentiu-se certa dificuldade em encontrar material que sustentasse a elaboração deste artigo, devido à escassez de produções que reúnam, em um só trabalho, exercício físico, exercício respiratório e doença de Parkinson.

No entanto, como aqui foi exposto, a dificuldade respiratória que atinge indivíduos diagnosticados com DP é pertinente, necessitando de maiores cuidados pelos profissionais que atuam diretamente com essa população. 


\section{Physical exercise as an instrument for improving breathing in Parkinson's disease: a case sudy}

\section{Abstract}

Parkinson's disease (PD) is a chronic, degenerative disease that affects primarily motor functions. The signs and symptoms of the disease are tremor, akinesia, muscular rigidity and postural instability. Due to the rigidity that leads to impairment of the flexor muscles of the trunk, the individual with PD shall present a reduced respiratory capacity. The objective of this study is to analyze the effects of physical exercise on physical and respiratory, respiratory and cognitive aspects in PD. This is a case study, lasting four months and collected pre, mid and post-intervention data. The volunteer was males, which should present complaining of difficulty breathing, clinically diagnosed with idiopathic PD, disease stage between mild to moderate, with no signs of dementia and level of physical activity considered active. As an initial interview and assessment will be conducted the following evaluations: clinical disease; cognitive; the physical and respiratory capacity. The intervention will consist of physical exercise, which the participant was already running, continuing at the same progressively adding and breathing exercises for two months, twice a week, one hour per session. After this time the revaluation will be performed using the same protocol. Two more months of intervention and the end of the final evaluation, which will total the four months of physical and breathing exercise program will be conducted. For data analysis, will be qualitative (clinical) and quantitatively (percentage).

Keywords: Parkinson's disease. Breathing. Muscular rigidity. Physical exercise.

\section{Referências}

ABRANTES, A. M. et al. Physical activity and neuropsychiatric symptoms of Parkinson's disease. Journal of Geriatric, Psychiatry and Neurology, Littleton, v. 25, n. 3, p. 138-145, Sept. 2012.

ALÓE, F. et al. Questionários de autoavaliação de sono. Escalas de avaliação clínica em psiquiatria e psicofarmacologia. São Paulo: Lemos, 2000. p. 423-434.

ALVES, L. A.; COELHO, A. C.; BRUNETTO, A. F. Fisioterapia respiratória na doença de Parkinson idiopática: relato de caso. $F i$ sioterapia e Pesquisa, São Paulo, v. 12, n. 3, p. 46-9, jun. 2005.

BARBIERI, F. A. et al. Functional capacity of Brazilian patients with Parkinson's disease (PD): Relationship between clinical characteristics and disease severity. Archives of Gerontology and Geriatrics, Amsterdam, v. 54, p. e83-e88, Mar.-Apr. 2012.

BARBOSA, E. R. Síndrome extrapiramidais. In: BACHESCHI, L. A.; NITRINI, R. A neurologia que todo médico deve saber. São Paulo: Santos, 1991. p. 223-238.

BERG, K. O. et al. Clinical and laboratory measures of postural balance in an elderly population. Archives of Physical Medicine and Rehabilitation, Philadelphia, v. 73, n. 11, p. 1073-1080, Nov. 1992.

BRAGA, A. et al. Benefícios do treinamento resistido na reabilitação da marcha $e$ equilíbrio nos portadores de doença de Parkinson. 2002. Disponível em: <http://www. saudeemmovimento.com.br/revista/artigos/ vida_e_saude/v2n2a9.pdf $>$. Acesso em: 20 nov. 2014 .

BROWN, R. G. et al. The Parkinson fatigue scale. Parkinsonism \& Related Disorders, Kidlington, v. 11, n. 1, p. 49-55, Jan. 2005. 
CALDEIRA, V. S. et al. Reliability and accuracy of cirtometry in healthy adults. Jornal Brasileiro de Pneumologia, São Paulo, v. 33, n. 5, p. 519-526, jan. 2007.

CAMARGOS, A. C. R. et al. O impacto da doença de Parkinson na qualidade de vida: uma revisão de literatura. Revista Brasileira de Fisioterapia, Belo Horizonte, v. 8, n. 3, p. 267-272, 2004.

CARRO, O. T. et al. Particularidades articulares de la disartria parkinsoniana. Revista Mexicana de Neurociência, Ciudad de Mexico, v. 2, n. 4, p. 235-239, abr. 2001.

COELHO, F. G. M. et al. Exercício físico no envelhecimento saudável e patológico. Curitiba: CRV, 2013. 468p.

COLE, R. J. Nonpharmacologic Techniques for Promoting Sleep. Clinics in Sports Medicine, Philadelphia, v. 24, p. 343-353, Apr. 2005.

CRUISE, K. E. et al. Exercise and Parkinson's: benefits for cognition and quality of life. Acta Neurologica Scandinavica, Copenhagen Munksgaard, v. 123, p. 13-19, Jan. 2011.

DUJARDIN, K.; LAURENT, B. Dysfunction of the human memory systems: role of the dopaminergic transmission. Current Opinion in Neurology, Philadelphia, v. 16, suppl. 2, p. S11-S16, Dec. 2003.

FOLSTEIN, M. F.; FOLSTEIN, S. E.; MCHUGH, P. R. Mini-mental state: a practical method for grading the cognitive state of patients for the clinician. Journal of Psychiatric Research, Oxford, v. 12, p. 189-198, Nov. 1975.

GOBBI, L. T. B. et al. Doença de Parkinson e exercício físico. Curitiba: CRV, 2014. 662p.

GOBBI, L. T. B. et al. Exercise programs improve mobility and balance in people with Parkinson's disease. Parkinsonism \& Related Disorders, Kidlington, v. 15 S3, p. S49-S52, Dec. 2009.
GONÇALVES, M. P. Influência de um programa de treinamento muscular respiratório no desempenho cognitivo e na qualidade de vida do idoso. 2007. 233f. Tese (Doutorado em Ciências da Saúde) - Faculdade de Ciências da Saúde, Universidade de Brasília, Brasília, 2007.

GORENSTEIN, C.; ANDRADE, L. Validation of a Portuguese version of the Beck depression inventory and the state-trait anxiety inventory in Brazilian subjects. Brazilian Journal of Medical and Biological Research, São Paulo, v. 29, p. 453-457, abr. 1996.

GRACE, J.; MENDELSOHN, A.; FRIEDMAN, J. H. A comparison of fatigue measures in Parkinson's disease. Parkinsonism \& Related Disorders, Kidlington, v. 13, n. 7, p. 443-445, Oct. 2007.

GRIMBERGEN, Y. A. et al. Postural instability in Parkinson's disease: the adrenergic hypothesis and the locus coeruleus. Expert Review of Neurotherapeutics, London, v. 9, n. 2, p. 279-290, Feb. 2009.

HERLOFSON, K.; LARSEN, J. P. Measuring fatigue in patients with Parkinson's disease - the Fatigue Severity Scale. European Journal of Neurology, Oxford, v. 9, n. 6, p. 595-600, Nov. 2002.

HOEHN, M. M.; YAHR, M. D. Parkinsonism: onset, progression and mortality. Neurology, Hagerstown, v. 17, n. 5, p. 427-442, May 1967.

HOVESTADT, A. et al. Pulmonary function in Parkinson's disease. Journal of Neurology, Neurosurgery \& Psychiatry, London, v. 52, p. 329-333, Mar. 1989.

KING, A. L. S. et al. A importância do foco da terapia cognitivo-comportamental direcionado às sensações corporais no transtorno do pânico: relato de caso. Revista de Psiquiatria Clínica, São Paulo, v. 34, n. 4, p. 191-195, mar. 2007. 
MONDOLO, F. et al. The validity of the hospital anxiety and depression scale and the geriatric depression scale in Parkinson's disease. Behavioural Neurology, London, v. 16 , p. 1-7, Oct. 2006.

MUOTRI, R. W.; NUNES, R. P.; BERNIK, M. A. Exercício aeróbio como terapia de exposição a estímulos interoceptivos no tratamento do transtorno do pânico. Revista Brasileira de Medicina do Esporte, São Paulo, v. 13, p. 327-330, set.-out. 2007.

NEVES NETO, A. R. Técnicas de respiração para a redução do estresse em terapia cognitivo-comportamental. Arquivos Médicos dos Hospitais e da Faculdade de Ciências Médicas da Santa Casa de São Paulo, São Paulo, v. 56, n. 3, p. 158-168, out. 2011.

O'SULLIVAN, S. B.; SCHMITZ, T. J. Fisioterapia: Avaliação e tratamento. 2. ed. São Paulo: Manole, 1993. 775p.

PARREIRA, V. F. et al. Padrão respiratório em pacientes portadores da doença de $\mathrm{Pa}$ rkinson e em idosos assintomáticos. Acta Fisiátrica, São Paulo, v. 10, n. 2, p. 61-63, ago. 2003.

PEREIRA, C. A. C. Espirometria. Jornal Brasileiro de Pneumologia, São Paulo, v. 28, n. S3, p. S1-S82, 2002.

PEREIRA, J. S.; CARDOSO, S. R. Distúrbio respiratório na doença de Parkinson. Fisioterapia Brasil, Rio de Janeiro, v. 1, n. 1, p. 23-26, set.-out. 2000 .

ROSA, J. C.; CIELO, C. A.; CECHELLA, C. Função fonatória em pacientes com Doença de Parkinson: uso de instrumento de sopro. Revista Cefac, Marília, v. 11, n. 2, p. 305-313, abr.-jun. 2009.

SABATÉ, M. et al. Obstructive and restrictive pulmonary dysfunction increases disability in Parkinson disease. Archives of Physical Medicine and Rehabilitation, Philadelphia, v. 77, p. 29-34, Jan. 1996.
SANTOS, F. A.; SOUZA, W. C.; BASTOS, V. H. A utilização do circuito de marcha como uma nova abordagem no tratamento da Doença de Parkinson (DP). Fisioterapia Ser, Rio de Janeiro, v. 2, n. 4, p. 279-283, 2007.

SCHULZ, G. M.; GRANT, M. K. Effects of speech therapy and pharmacologic and surgical treatments on voice and speech in Parkinson's disease: a review of the literature. Journal of Communication Disorders, Amsterdam, v. 33, n. 1, p. 59-88, 2000.

SEREBROVSKAYA, T. et al. Hypoxic ventilatory responses and gas exchange in patients with Parkinson's disease. Respiration, New York, v. 65, p. 28-33, 1998.

SOCIEDADE BRASILEIRA DE CARDIOLOGIA. Diretriz de reabilitação cardíaca. Arquivos Brasileiros de Cardiologia, São Paulo, v. 84, n. 5, p. 431-440, maio 2005.

SPREEN, O.; STRAUSS, E. A compendium of neuropsychological tests: administration, norms and commentary. 2. ed. New York: Oxford University Press, 1998. 752p.

TANAKA, K. Influência do exercício físico em memória e funções executivas de pessoas com Doença de Parkinson. 2008. 92f. Dissertação (Mestrado em Biodinâmica da Motricidade Humana) - Faculdade de Educação Física, Universidade Estadual Paulista, Rio Claro, 2008.

TANAKA, K. et al. Benefits of physical exercise on executive functions in older people with Parkinson's disease. Brain and Cognition, New York, v. 69, p. 435-441, Mar. 2009.

TEKIN, S.; CUMMINGS, J. L. Frontalsubcortical neuronal circuits and clinical neuropsychiatry: an update. Journal of Psychosomatic Research, Oxford, v. 53, n. 2, p. 647-654, Aug. 2002.

VITÓRIO, R. et al. Effects of 6-month, multimodal exercise program on clinical and gait parameters of patients with idiopathic Parkinson's disease: a pilot study. ISRN Neurology, Cairo, v. 2011, p. 1-7, Oct. 2011. 
VOORRIPS, L. E. et al. A physical activity questionaire for the elderly. Medicine and Science in Sports and Exercise, Hagerstown, v. 23, n. 8, p. 974-979, Aug. 1991.

WERMUTH, L. et al. Mortality in patients with Parkinson's disease. Acta Neurologica Scandinavica, Copenhagen Munksgaard, v. 92, p. 55-58, July 1995.

HOPKINS, M. E. et al. Differential effects of acute and regular physical exercise on cognition and affect. Neuroscience, Oxford, n. 215 , p. $59-68$, jul. 2012. 